\title{
Rational Use of Tocilizumab in the Treatment of Novel Coronavirus Pneumonia
}

\author{
Shengyu Zhang ${ }^{1} \cdot$ Lei Li $^{2} \cdot$ Aizong Shen ${ }^{1} \cdot$ Yongwu Chen ${ }^{1} \cdot$ Zhigang $i^{3}$
}

Published online: 26 April 2020

C) Springer Nature Switzerland AG 2020

\begin{abstract}
Since December 2019, a novel coronavirus pneumonia (COVID-19) has broken out in Wuhan, China and spread rapidly. Recent studies have found that $\sim 15.7 \%$ of patients develop severe pneumonia, and cytokine storm is an important factor leading to rapid disease progression. Currently, there are no specific drugs for COVID-19 and the cytokine storm it causes. IL-6 is one of the key cytokines involved in infection-induced cytokine storm. Tocilizumab, which is the IL-6 receptor antagonist, has been approved by the US FDA for the treatment of cytokine release syndrome (CRS), is expected to treat cytokine storm caused by COVID-19 and is now in clinical trials. In this paper, we will elaborate the role of cytokine storm in COVID-19, the mechanism of tocilizumab on cytokine storm and the key points of pharmaceutical care based on the actual clinical application for COVID-19 in our hospital, the latest research reports, clinical trial progress of tocilizumab, drug instruction from the US FDA, and "Diagnosis and Treatment Plan of Novel Coronavirus Pneumonia (seventh trial edition)" in China, so as to provide reference for the treatment of COVID-19.
\end{abstract}

\section{Key Points}

Cytokine storm is an important factor in the rapid deterioration of patients with COVID-19.

Tocilizumab, an IL-6 receptor antagonist, is expected to be used in the treatment of cytokine storm caused by COVID-19 and is recommended as an immunotherapy drug for critical COVID-19 patients in China.

Tocilizumab should be used cautiously in patients with serious infections, neutropenia, thrombocytopenia, and liver damage.

Zhigang Qi

qizhigang058518@163.com

1 Department of Pharmacy, The First Affiliated Hospital of University of Science and Technology of China, Hefei 230001, Anhui, China

2 Infection Department, The First Affiliated Hospital of University of Science and Technology of China, Hefei 230001, Anhui, China

3 Department of Pharmacy, Wuxi People's Hospital Attached to Nanjing Medical University, Wuxi 214023, Jiangsu, China

\section{Introduction}

In December 2019, coronavirus disease 2019 (COVID-19) caused by severe acute respiratory syndrome coronavirus 2 (SARS-CoV-2) was first identified in Wuhan, China, and has spread rapidly in many countries. The team of Nanshan Zhong found that $87.9 \%$ patients had fever after hospitalization and in $15.7 \%$ patients this developed to severe pneumonia [1]. When COVID-19 progresses from severe to critical, patients may develop severe cytokine storm, secondary acute respiratory distress syndrome, followed by shock, tissue perfusion disorders, and even multi-organ failure [2]. Cytokine storm is an important factor in the rapid progression of COVID-19. Therefore, the treatment of cytokine storm is an important part of rescuing severe patients. Currently, there is no specific drug for SARS-CoV-2 and cytokine storm induced by COVID-19. The IL-6 receptor antagonist tocilizumab has been approved by the US FDA for the treatment of cytokine release syndrome (CRS). Since IL-6 is one of the key cytokines involved in infection-induced cytokine storm, the treatment of cytokine storm induced by COVID-19 with tocilizumab has broad prospects. "Diagnosis and Treatment Plan of Novel Coronavirus Pneumonia (seventh trial edition)" in China recommends tocilizumab as an immunotherapy drug for critical patients [3]. However, the clinical experience and data of tocilizumab in the 
treatment of COVID-19 are limited. In this paper, several authors who participated in the treatment and consultation of critical and difficult patients and had specific experience in the clinical application of tocilizumab will explain the role of cytokine storm in COVID-19, the mechanism of tocilizumab in treatment of COVID19 and the key points of pharmaceutical care according to the latest research reports and clinical trial progress, so as to provide reference for the treatment of COVID-19.

\section{The Role of Cytokine Storm in COVID-19}

Cytokines, which are a group of small proteins secreted by cells and used mainly for inter-cell signaling and communication, have a variety of biological functions such as regulating innate immunity, adaptive immunity, hematopoiesis, cell growth and differentiation, and repairing damaged tissues by binding to receptors [4]. Cytokines mainly include interleukin (IL), interferon (IFN), tumor necrosis factor (TNF), colony stimulating factor (CSF), chemokine and growth factor. When the human body is invaded by bacteria and viruses, the immune system will release a large number of cytokines $[5,6]$. In this outbreak of COVID-19, some infected patients would suddenly worsen in the later stage and eventually die from multiple organ failure, and the aggravation was mainly caused by cytokine storm [7].

It can be considered that cytokine storm is a kind of over-immune phenomenon produced by the body in response to external stimuli and then an uncontrolled release of cytokines will lead to systemic inflammation. In 1993, Ferrara et al. first proposed the concept of cytokine storm in graft-versus-host disease [8]. Then in 2003 after SARS, the concept of cytokine storm gradually emerged due to the extremely high mortality associated with multi-organ failure [9]. In recent years, chimeric antigen receptor $\mathrm{T}$ (CAR-T) cells, as immunotherapy, have used transgenic $\mathrm{T}$ cells to attack cancer cells, and have shown remarkable efficacy against previously refractory malignancies. However, patients treated with CAR-T cells produce many potentially life-threatening toxic reactions, and one of the most clinically significant toxic reaction is CRS. The clinical symptoms of CRS involve multiple organ systems, with life-threatening complications including fluid-refractory hypotension and cardiac dysfunction, respiratory failure, coagulopathy, renal and liver failure. CRS occurs because of the high level of immune activation of lymphocytes, macrophages, or myeloid cells with a subsequent massive release of inflammatory cytokines. In CAR-T cell-related CRS, the marked elevation of IL-6 is considered to be one main factor of the symptoms. The level of IL- 6 and IFN- $\gamma$ increase after CAR-T treatment and then stimulate the differentiation of $\mathrm{T}$ helper (Th) 17 cells, inducing immunologic response and the development of CRS [10]. In many cases, cytokine storm and CRS are the same concept [11].

Previous therapy for controlling cytokine storm with large doses of glucocorticoid may delay the clearance of coronavirus and cause complications of glucocorticoid therapy [12]. Therefore, it is urgent to find new and specific drug treatment methods.

\section{Tocilizumab and its Mechanism of Action in the Treatment of COVID-19}

A data analysis of 138 hospitalized COVID-19 patients was published in JAMA on February 7, 2020 [13], and the course of disease was revealed for the first time in the deceased patients. Neutrophil counts, D-dimer levels, blood urea nitrogen and creatinine levels continued to increase in the dying patients, while lymphocyte counts continued to decrease. It was suggested that the virus induced an increase in the number of neutrophils caused by cytokine storm, leading to clotting activation, hypoxia, shock, and acute kidney injury. These pathological mechanisms may be closely relevant to the death of COVID-19 patients [14]. A number of recently published articles have analyzed the clinical characteristics of COVID-19 patients, and consistently found that the lymphocyte count was significantly reduced in patients with pneumonia, especially those with severe pneumonia, while numerous cytokines (such as IL-6, IL-10, IL-2 and TNF and IFN- $\gamma$ ) were significantly increased, and pointed out that the high levels of IL-6 and other cytokines in vivo were the main cause of the cytokine storm [13-17]. Jing et al. studied the lymphocyte subsets and cytokines in peripheral blood of 40 patients with COVID-19, and found that the T cell count and cytokine levels of the survivors with severe infection gradually recovered to the same level as those with mild infection in the following time points [17]. As an inflammatory trigger IL-6 was highly expressed in patients with COVID-19, and could induce the differentiation of B lymphocytes and the production of antibodies, and also participate in the immune response of the body by inducing the proliferation and differentiation of T lymphocytes [18]. The team of Haiming Wei showed that after the SARS-CoV-2 infection, $\mathrm{CD} 4^{+} \mathrm{T}$ lymphocytes were rapidly activated to become pathogenic Th 1 cells and generate GM-CSF, etc. The cytokines environment induced inflammatory $\mathrm{CD} 14^{+} \mathrm{CD} 16^{+}$monocytes with high expression of IL- 6 and accelerated the inflammation. These aberrant and excessive immune cells might enter the pulmonary circulation in huge numbers and play an immune damaging role in leading to lung functional disability and quick mortality [19]. IL-6 is also considered to be a key mediator of CRS. Thus, targeted drugs that inhibit IL- 6 can block CRS or cytokine storm [20]. 
As a monoclonal antibody that can antagonize the IL-6 receptor, tocilizumab has promising prospects. Based on the efficacy of several studies on CRS [21,22], tocilizumab is currently approved by the US FDA for the treatment of severe CRS in addition to idiopathic arthritis, rheumatoid arthritis, and giant cell arteritis. Significantly, "Diagnosis and Treatment Plan of Novel Coronavirus Pneumonia (seventh trial edition)" in China [3] recommends that tocilizumab can be used in patients with extensive bilateral lung lesions and severe lung diseases, who have elevated IL-6 levels in laboratory tests.

The mechanism of tocilizumab in the treatment of COVID-19 remains unclear. According to previous studies, IL-6 is secreted by almost all stromal cells and immune system cells, such as B lymphocytes, T lymphocytes, macrophages, monocytes, dendritic cells, mast cells and other non-lymphocytes, such as fibroblasts, endothelial cells, keratinocytes, glomerular mesangial cells and tumor cells [23]. Under normal circumstances, the level of IL-6 in the body is very low, and it can be quickly synthesized to strengthen the body's defense function when there is an infection or injury. Excessive release of IL-6 can cause CRS, and the more severe the CRS, the higher the serum peak concentration of IL-6. IL-6 binds to its receptor IL-6R to form a complex, and then binds to the signal transducer glycoprotein 130 (gp-130) to initiate signal transduction and trigger downstream signal transduction and gene expression. IL-6R exists not only in membrane-bound form (mIL-6R), but also in soluble form (sIL-6R). In the classical signal transduction pathway, IL-6 binds to mIL-6R to form a complex, and then binds to gp-130, causing downstream reactions, such as anti-inflammatory effects, that are limited to such cells that express mIL-6R. In the trans-signaling pathway, IL-6 forms a complex with sIL-6R and gp-130, which initiates intracellular signal transduction in the absence of mIL-6R, and the pathway has inflammatory effects [24]. The next steps were to induce the synthesis of acute reactive protein through two completely different signaling pathways. One IL-6 signaling pathway is mediated by the JAK/STAT tyrosine kinase system, while the other is mediated by the Ras/mitogenactivated protein kinase (MAPK)/NF-KB-IL-6 pathway. The former is a major pathway [25, 26].

In the classical signal pathway, many cells cannot respond to IL-6 signal because of the lack of expression of IL-6R, while some of these cells can be stimulated by sIL-6R-IL-6 complex to respond to IL-6 signal and cause cell signal transduction [25, 27]. The trans-presentation signal is suppressed by extracellular gp-130, and extracellular gp- 130 can form a complex with sIL-6R to prevent sIL-6R from binding to membrane-bound gp-130 [23].

The classical signal is limited to the cells (macrophages, neutrophils, $\mathrm{T}$ cells, etc.) that express IL-6R, and plays a leading role in the low level of IL-6. However, when the level of IL-6 increases, IL-6 signal is widely expressed, because gp-130 is everywhere. In this way, IL-6 trans signaling via the sIL-6R can activate virtually all cells of the body and then regulate pro-inflammatory reactions. Blocking of trans-signaling was effective in a variety of preclinical chronic and autoimmune disease models [28]. Tocilizumab, which is a humanized anti-IL-6R monoclonal antibody, can bind to both mIL-6R and sIL-6R and then inhibit classical and trans-signals. This may be its potential mechanism for the treatment of cytokine storm in COVID19 (see Fig. 1).

\section{Progress in Clinical Trials of Tocilizumab in the Treatment of COVID-19}

To date, several independent clinical trials have been initiated globally to explore the efficacy and safety of tocilizumab for the treatment of patients with COVID-19.

A prospective multicenter randomized controlled clinical study led by the first affiliated hospital of university of science and technology of China has been registered in the Chinese clinical trial registry. This study included 188 patients with common type (including critical risk factors) and severe COVID-19 and is expected to be completed in May 2020 [29] (see Table 1).

In the first phase of the study, all 14 COVID-19 patients (the oldest patient was aged 82 years, including 9 severe patients and 2 critical patients) had diffuse lesions in both lungs before treatment, and 11 had persistent fever. After treatment with the new regimen of tocilizumab combined with conventional therapy, the body temperature of all 11 patients with fever dropped to normal within $24 \mathrm{~h}$. Meanwhile, the respiratory function and oxygenation index were improved in different degrees. CT scan showed obvious improvement of pulmonary lesions in 4 patients. Therefore, for patients with severe COVID-19 caused by cytokine storm, tocilizumab is a drug worthy of clinical research.

In addition, Genentech, a member of the Roche Group, announced that the US FDA has approved a Phase III clinical trial for tocilizumab to treat severe covid-19 pneumonia on March 23, 2020. This is a randomized, double-blind, placebo-controlled Phase III study to evaluate the safety and efficacy of intravenous tocilizumab added to standard of care in adult patients hospitalized with severe COVID19 pneumonia compared to placebo plus standard of care. The primary and secondary endpoints include clinical status, mortality, mechanical ventilation and ICU variables. Patients will be followed up for 60 days post-randomization, and an interim analysis will be conducted to look for early evidence of efficacy [30]. 
Fig. 1 The potential mechanism of tocilizumab in the treatment of cytokine storm induced by COVID-19 SARS-CoV-2 severe acute respiratory syndrome coronavirus 2; IL interleukin, $M A P K$ mitogen-activated protein kinase

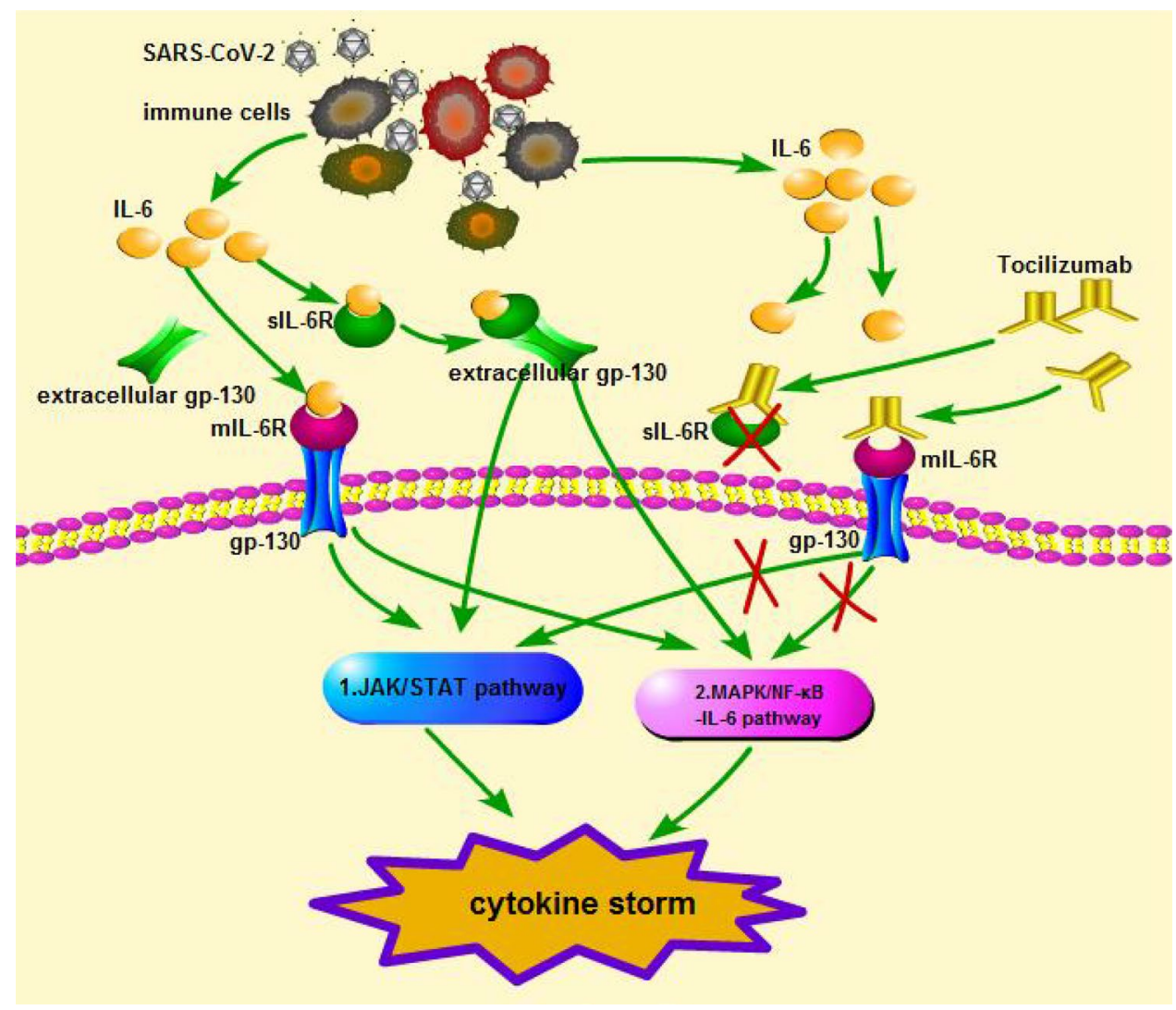

Table 1 A multicenter, randomized controlled trial of tocilizumab in the treatment of COVID-19 [29]

Items Details

Registration number

Date of registration

Public title

Applicant's institution

Approved No. of ethic committee

Inclusion criteria

Exclusion criteria

\section{ChiCTR2000029765}

2020-02-13

A multicenter, randomized controlled trial for the efficacy and safety of tocilizumab in the treatment of new coronavirus pneumonia (COVID-19)

The First Affiliated Hospital of University of science and technology of China

2020-XG(H)-008: The First Affiliated Hospital of University of science and technology of China Ethics committee

1. The patients who were diagnosed with the common type of NCP (including severe risk factors) and severe cases of new coronavirus pneumonia

2. Aged $18-85$ years

3. IL-6 elevated (using Elisa method, using the same company kit)

4. Patients or authorized family members volunteered to participate in this study and signed informed consent Definition of Novel Coronavirus Pneumonia (NCP) clinical cases: Regular patients with NCP (including severe risk factors): patients with dual pulmonary lesions based on common NCP clinical symptoms accompanied by fever or no fever; Critical NCP patient: Refer to the "New Coronavirus Pneumonia Diagnosis and Treatment Plan (Fifth Edition)" formulated by the National Health Commission

Patients who are participating in other drug clinical trials; Pregnant or lactating women; ALT/AST $>5$ ULN, neutrophils $<0.5 \times 10^{9} / \mathrm{L}$, platelets less than $50 \times 10^{3} / \mu \mathrm{L}$; Definite diagnosis of rheumatic immune-related diseases; Long-term oral anti-rejection or immunomodulatory drugs; Hypersensitivity to tocilizumab or any excipients; Patients with active pulmonary tuberculosis, with definite bacterial and fungal infections

$A L T$ alanine aminotransferase; $A S T$ aspartate aminotransferase; $U L N$ upper limit of normal 


\section{Clinical Application of Tocilizumab}

A research team from University of Science and Technology of China immediately carried out a small clinical study in the first affiliated hospital (our hospital) and Anhui Fuyang second people's hospital after discovering the mechanism of cytokine storm in COVID-19 patients. During the period from February 5 to 14, the COVID-19 patients diagnosed as severe or critical in these two hospitals were given tocilizumab in addition to routine therapy. Within a few days, the fever returned to normal and all other symptoms improved remarkably. By the time their paper was published, 15 patients (75.0\%) had lowered their oxygen intake and 1 patient no longer needed oxygen therapy. CT scans manifested that the lung lesion opacity absorbed in 19 patients (90.5\%). The percentage of lymphocytes in peripheral blood, which decreased in $85.0 \%$ patients (17/20) before treatment (mean, $15.52 \pm 8.89 \%$ ), returned to normal in $52.6 \%$ patients $(10 / 19)$ on the fifth day after treatment. Abnormally elevated C-reactive protein decreased significantly in $84.2 \%$ patients $(16 / 19)$. No obvious adverse reactions were observed. Nineteen patients $(90.5 \%)$ have been discharged on average 13.5 days after the treatment with tocilizumab and the rest are recovering well [31].

\subsection{Patient Selection and Administration}

\subsubsection{Recommendation of Drug Instruction from the US FDA}

Tocilizumab is recommended for adults and pediatric patients aged $\geq 2$ years with CAR-T cell-induced severe or life-threatening CRS to be used alone or in combination with glucocorticoids (see Table 2 for specific usage and dosage [32]).

Doses exceeding $800 \mathrm{mg}$ per infusion are not recommended in CRS patients. The duration of a single intravenous drip should be maintained for more than $1 \mathrm{~h}$. Subcutaneous administration is not recommended for the treatment of CRS. If no clinical improvement in the signs

Table 2 Dosage and administration for CRS

\begin{tabular}{lll}
\hline Weight $(\mathrm{kg})$ & Single dose $(\mathrm{mg} / \mathrm{kg})$ & \begin{tabular}{l} 
Intravenous formulation \\
\hline$<0$
\end{tabular} \\
& 12 & $\begin{array}{l}\text { Dilute to } 50 \mathrm{~mL} \text { in } 0.9 \% \\
\text { or } 0.45 \% \text { sodium } \\
\text { chloride injection }\end{array}$ \\
& & Dilute to $100 \mathrm{~mL}$ \\
& 8 & in $0.9 \%$ or $0.45 \%$ \\
& & sodium chloride \\
& & injection
\end{tabular}

$C R S$ cytokine-release syndrome and symptoms of CRS occurs after the first dose, up to 3 additional doses of tocilizumab may be administered. The interval between consecutive doses should be at least $8 \mathrm{~h}$.

\subsubsection{Recommendation of Chinese COVID-19 Diagnosis and Treatment Plan}

Tocilizumab has not been approved for CRS in China. However, "Diagnosis and Treatment Plan of Novel Coronavirus Pneumonia (seventh trial edition)" in China [3] recommended that tocilizumab could be used in patients with extensive lung lesions and severe diseases, and those with high IL-6 levels in laboratory tests. For the first dose of $4-8 \mathrm{mg} / \mathrm{kg}$, the recommended dose is $400 \mathrm{mg}$, diluted to $100 \mathrm{~mL}$ with $0.9 \%$ sodium chloride injection, and the infusion time is more than $1 \mathrm{~h}$. For patients with poor efficacy of the first dose, an additional dose (the dose is the same as before) can be applied after $12 \mathrm{~h}$, with a maximum of 2 cumulative doses and a maximum of $800 \mathrm{mg}$ for a single dose.

\subsection{Drug Safety Monitoring}

Since its introduction, tocilizumab has shown good efficacy and safety in the treatment of a variety of autoimmune diseases. Ferfar et al. [33] summarized a number of literatures on tocilizumab treatment of Takayasu arteritis and found that its use could reduce (or even stop) the dosage of glucocorticoids. Samson et al. [34] prospectively observed 20 patients with giant cell arteritis treated with prednisone combined with tocilizumab $(8 \mathrm{mg} / \mathrm{kg}$, intravenous infusion once every 4 weeks), and confirmed the same findings at 52 weeks of follow-up.

A randomized, double-blind, Phase III clinical study showed that 18 patients with Takayasu arteritis treated with tocilizumab did not experience serious infections and deaths, nor did they have new and more prominent safety issues compared with the placebo group [35]. Ferfar et al. [33] reviewed the use of tocilizumab to treat 38 patients with Takayasu arteritis, including 2 cases of liver injury, 2 cases of infection, and 4 cases of leukopenia, and the incidence of adverse drug reactions reached $21 \%$. This is consistent with the results of a Chinese study on the safety of tocilizumab in the treatment of Takayasu arteritis [36]. However, these studies are small in sample size and need to be further confirmed by multicenter large sample studies.

Based on the above studies, drug instructions of China and the US FDA, the following adverse reactions need to be noted. (1) Serious infections: The most common serious infections included pneumonia, urinary tract infection, cellulitis, herpes zoster, gastroenteritis, diverticulitis, sepsis and bacterial arthritis. (2) Gastrointestinal perforations: Gastrointestinal perforations were primarily reported as 
complications of diverticulitis including generalized purulent peritonitis, lower GI perforation, fistula and abscess. Most patients who developed gastrointestinal perforations were taking concomitant nonsteroidal anti-inflammatory drugs (NSAIDs), corticosteroids, or methotrexate at the same time. (3) Infusion reactions: The most frequently reported event on the $4 \mathrm{mg} / \mathrm{kg}$ and $8 \mathrm{mg} / \mathrm{kg}$ dose during the infusion was hypertension, while the most frequently reported events occurring within $24 \mathrm{~h}$ after infusion were headache and skin reactions. These events were not treatment limiting. (4) Anaphylaxis: These reactions were generally observed during the second to fourth infusion of tocilizumab. (5) Abnormal laboratory indexes: Such as thrombocytopenia, elevated liver enzymes and elevated lipid parameters (total cholesterol, LDL, triglycerides).

The above adverse reactions data were obtained from a series of randomized controlled clinical studies of longcourse treatment with tocilizumab. However, whether the adverse reactions will occur during the short-course treatment of COVID-19 needs to be further demonstrated.

For all these reasons, the safety monitoring of tocilizumab mainly includes the following three points: (1) Risk of serious infections including tuberculosis, bacteria, invasive fungi, viruses, and other opportunistic infections, leading to hospitalization or death. If severe infection occurs, tocilizumab should be discontinued until the infection is under control. Perform a latent tuberculosis test and, if positive, start treating tuberculosis before starting tocilizumab. Monitor active tuberculosis for all patients during treatment. (2) Tocilizumab should be used with caution in patients with absolute neutrophil count (ANC) $<2 \times 10^{9} / \mathrm{L}$, and is not recommended for patients with $\mathrm{ANC}<0.5 \times 10^{9} / \mathrm{L}$. Tocilizumab should be used with caution in patients with a platelet count less than $100 \times 10^{3} /$ $\mu \mathrm{L}$, and is not recommended for patients with platelet counts less than $50 \times 10^{3} / \mu \mathrm{L}$. (3) Care should be taken when starting tocilizumab in patients whose ALT or AST rises above the 1.5-fold upper limit of normal. For patients whose ALT or AST rises above the 5-fold upper limit of normal, tocilizumab is not recommended.

\subsection{The Use of Tocilizumab in Special Populations}

The following information is mainly from the drug instructions of China and the US FDA.

Pregnant women It is known that tocilizumab actively crosses the placenta in late pregnancy and may affect the fetal immune response. It is not yet possible to predict drug-related birth defects and the risk of miscarriage. In animal reproduction studies [32], intravenous administration of tocilizumab during organogenesis in cynomolgus monkeys may result in miscarriage or stillbirth at doses higher than the maximum recommended dose of intravenous administration of $8 \mathrm{mg} / \mathrm{kg}$ every $2-4$ weeks. Animal experiments have shown that inhibition of the IL-6 signal may interfere with cervical maturation, expansion and contraction of the uterine muscle, which may be potentially dangerous to the fetus [32].

Older adults Current studies suggest that patients aged $\geq 65$ years treated with tocilizumab have a higher rate of severe infection than patients $\leq 65$ years. Due to the higher infection rate of the elderly in general, care should be taken in their treatment [32].

Patients with liver damage There is a lack of pharmacokinetic data on the use of tocilizumab in patients with liver damage.

Patients with renal impairment Mild renal impairment (creatinine clearance $<80 \mathrm{~mL} / \mathrm{min}$ and $\geq 50 \mathrm{~mL} / \mathrm{min}$ calculated by the Cockcroft-Gault formula) did not affect the pharmacokinetics of tocilizumab. In clinical trials of giant cell arteritis, approximately one-third of patients had moderate renal impairment at baseline (creatinine clearance was between 30 and $59 \mathrm{~mL} / \mathrm{min}$ ), and no effect on tocilizumab exposure was found in these patients [32].

\section{Conclusion}

It has been confirmed by the recent reports on the clinical characteristics of COVID-19 and the mechanism of disease progression that cytokine storm played a key role in the rapid progress of the disease. IL-6 is one of the key cytokines involved in infection-induced cytokine storm. Tocilizumab can block the downstream signal transduction by binding mIL-6R and sIL-6R and play a role in the treatment of cytokine storm caused by COVID19. Currently, the usage and dosage of tocilizumab for COVID-19 are still being explored. The clinical use of tocilizumab can be referred to drug instruction from the US FDA for the treatment of CRS or "Diagnosis and Treatment Plan of Novel Coronavirus Pneumonia (seventh trial edition)" in China. Be aware of the risk of serious infection, neutropenia or thrombocytopenia, and liver damage caused by tocilizumab. There is no need to adjust the dose of tocilizumab in patients with mild or moderate renal insufficiency, and the data of using tocilizumab in pregnant women and patients with liver injury are insufficient.

Author Contributions LL, ZQ were involved in the design of the review. YC collected the literature. SZ and ZQ wrote the first draft of the manuscript, which was critically revised by AS. 


\section{Compliance with Ethical Standards}

Funding The preparation of this review was supported by Wuxi Medical and Health Guidance Plan for scientific and technological development [Grant numbers NZ2019004].

Conflict of interest Shengyu Zhang, Lei Li, Aizong Shen, Yongwu Chen and Zhigang Qi declare that they have no conflict of interest.

\section{References}

1. Guan WJ, Ni ZY, Hu Y, et al. Clinical characteristics of 2019 novel coronavirus infection in China. MedRxiv. 2019;2020:2020. https://doi.org/10.1101/2020.02.06.20020974. Accessed $20 \mathrm{Feb}$ 2020.

2. Xu KJ,Cai HL,Shen YH,et a1. Management of corona virus disease-19 (COVID-19): the Zhejiang experience. Zhejiang Da Xue Xue Bao (Yi Xue Ban). 2020. https://kns.cnki.net/kcms/detai 1/33.1248.R.20200222.1417.002.html. Accessed 3 Mar 2020 (in Chinese).

3. General Office of the National Health Committee of China, China Traditional Chinese Medicine Administration Office. Diagnosis and treatment plan of novel coronavirus pneumonia (seventh trial edition). 2020. http://www.nhc.gov.cn/yzygj/s7652m/202003/ a31191442e29474b98bfed5579d5af95.shtml. Accessed 11 Mar 2020.

4. Jennifer RT, Marcus JK, Cameron PS, et al. Into the eye of the cytokine storm. Microbiol Mol Biol Rev. 2012;76(1):16-32.

5. Fei ZY, Dong H, Qian QJ, et al. Analysis of pathogens and risk factors for death in elderly patients with severe pneumonia. Zhong Hua Yi Yuan Gan Ran Xue Za Zhi. 2019;29(3):380-3 (in Chinese).

6. Ma YP, Yan YH, Sun XD, et al. Correlation between inflammatory factor expression level in severe pneumococcal alveolar lavage fluid and prognosis. Zhong Hua Yi Yuan Gan Ran Xue Za Zhi. 2019;29(7):1007-10 (in Chinese).

7. Zhang JW, Hu X, Jin PF. Cytokine storm induced by SARS-CoV-2 and the drug therapy. Zhongguo Yao Xue Za Zhi. 2020. https ://kns.cnki.net/kcms/detail/11.2162.R.20200225.1052.002.html. Accessed 9 Feb 2020 (in Chinese).

8. Ferrara JL, Abhyankar S, Gilliland DG. Cytokine storm of graftversus-host disease: a critical effector role for interleukin-1. Transplant Proc. 1993;25(1 Pt 2):1216-17.

9. De Jong MD, Simmons CP, Thanh TT, et al. Fatal outcome of human influenza $\mathrm{A}(\mathrm{H} 5 \mathrm{~N} 1)$ is associated with high viral load and hypercytokinemia. Nat Med. 2006;12(10):1203-7.

10. Teachey DT, Lacey SF, Shaw PA, et al. Identification of predictive biomarkers for cytokine release syndrome after chimeric antigen receptor T-cell therapy for acute lymphoblastic leukemia. Cancer Discov. 2016;6(6):664-79.

11. Zhang YL, Jiang CY. Cytokine storm in ARDS. Sheng Ming Ke Xue. 2015;5:554-7 (in Chinese).

12. Russell CD, Millar JE, Baillie JK. Clinical evidence does not support corticosteroid treatment for 2019-nCoV lung injury. Lancet. 2020;395(10223):473-5.

13. Wang $\mathrm{D}, \mathrm{Hu} \mathrm{B}, \mathrm{Hu} \mathrm{C}$, et al. Clinical Characteristics of 138 Hospitalized Patients With 2019 Novel Coronavirus-Infected Pneumonia in Wuhan, China. JAMA. 2019;2020:2020. https:// doi.org/10.1001/jama.2020.1585. Accessed 15 Feb 2020.

14. Huang CL, Wang YM, Li XW, et al. Clinical features of patients infected with 2019 novel coronavirus in Wuhan, China. Lancet. 2020;395(10223):497-506
15. Chen L, Liu HG, Liu W, et al. Analysis of clinical features of 29 patients with 2019 novel coronavirus pneumonia. Zhong Hua Jie He He Hu Xi Za Zhi. 2019;2020:2020. https://doi.org/10.3760/ cma.j.issn.1001-0939.2020.0005. Accessed 15 Feb 2020 (in Chinese) .

16. Wan SX, Yi QJ, Fan SB, et al. Characteristics of lymphocyte subsets and cytokines in peripheral blood of 123 hospitalized patients with 2019 novel coronavirus pneumonia (NCP). MedRxiv. 2019;2020:2020. https://doi.org/10.1101/2020.02.10.20021832. Accessed 20 Feb 2020.

17. Jing L, Li S, Liu J, et al. Longitudinal characteristics of lymphocyte responses and cytokine profiles in the peripheral blood of SARS-CoV-2 infected patients. MedRxiv. 2020. https://doi. org/10.1101/2020.02.16.20023671. Accessed 3 Mar 2020.

18. Zhai H, Liu YW, Wang Y, et al. The pathogen distribution and its influence on inflammatory factors in old patients with heart failure complicated with pulmonary infection. Tianjin Yi Yao. 2018;46(9):952-5 (in Chinese).

19. Zhou YG, Fu BQ, Zheng XH, et al. Aberrant pathogenic GM-CSF $+\mathrm{T}$ cells and inflammatory CD14 + CD16 + monocytes in severe pulmonary syndrome patients of a new coronavirus. BioRxiv. 2020. https://doi.org/10.1101/2020.02.12.945576. Accessed 20 Mar 2020.

20. Tanaka T, Narazaki M, Kishimoto T. Immunotherapeutic implications of IL-6 blockade for cytokine storm. Immunotherapy. 2016;8(8):959-70.

21. Fitzgerald JC, Weiss SL, Maude SL, et al. Cytokine release syndrome after chimeric antigen receptor $\mathrm{T}$ cell therapy for acute lymphoblastic leukemia. Crit Care Med. 2017;45:e124-31.

22. Maude SL, Laetsch TW, Buechner J, et al. Tisagenlecleucel in children and young adults with B-cell lymphoblastic leukemia. N Engl J Med. 2018;378(5):439-48.

23. Jones SA, Jenkins BJ. Recent insights into targeting the IL-6 cytokine family in inflammatory diseases and cancer. Nat Rev Immunol. 2018;18(12):773-89.

24. Braun GS, Nagayama Y, Maruta Y, et al. IL-6 trans-signaling drives murine crescentic GN. Am Soc Nephrol. 2016;27(1):132-42.

25. Villarino AV, Kanno Y, O'Shea JJ. Mechanisms and consequences of Jak-STAT signaling in the immune system. Nat Immunol. 2017;18(4):374-84.

26. Zegeye MM, Madelene L, Knut F, et al. Activation of the JAK/ STAT3 and PI3K/AKT pathways are crucial for IL-6 trans-signaling-mediated pro-inflammatory response in human vascular endothelial cells. Cell Commun Signal. 2018;16(1):55.

27. Johnson DE, O'Keefe RA, Grandis JR. Targeting the IL-6/ JAK/STAT3 signalling axis in cancer. Nat Rev Clin Oncol. 2018;15(4):234-48.

28. Rose-John S. The soluble Interleukin 6 receptor: advanced therapeutic options in inflammation. Clin Pharmacol Ther. 2017;102(4):591-8.

29. The first affiliated hospital of university of science and technology. The efficacy and safety of tocilizumab in the treatment of novel coronavirus pneumonia: a multi-center, randomized, double-blinded trial. 2020. https://www.chictr.org.cn/showproj. aspx?proj=49409. Accessed 22 Feb 2020.

30. Genentech. Genentech announces FDA approval of clinical trial for actemra to treat hospitalized patients with severe COVID19 Pneumonia;. 2020. https://www.gene.com/media/press-relea ses/14843/2020-03-23/genentech-announces-fda-approval-of-clin. Accessed 27 Mar 2020

31. Xu XL, Han MF, Li TT, et al. Effective Treatment of Severe COVID-19 Patients with Tocilizumab. ChinaXiv. 2020. http:// www.chinaxiv.org/abs/202003.00026. Accessed 20 Mar 2020. 
32. The U.S. Food and Drug Administration. Information for ACTEMRA $^{\circledR}$ (tocilizumab). https://www.accessdata.fda.gov/ drugsatfda_docs/label/2017/125276s114lbl.pdf. Accessed 15 Feb 2020.

33. Ferfar Y, Mirault T, Desbois AC, et al. Biotherapies in large vessel vasculitis. Autoimmun Rev. 2016;15(6):544-51.

34. Samson M, Devilliers H, Ly KH, et al. Tocilizumab as an addon therapy to glucocorticoids during the first 3 months of treatment of Giant cell arteritis: a prospective study. Eur J Intern Med. 2018;57:96-104.
35. Nakaoka Y, Isobe M, Takei S, et al. Efficacy and safety of tocilizumab in patients with refractory Takayasu arteritis: results from a randomised, double-blind, placebo-controlled, phase 3 trial in Japan(the TAKT study). Ann Rheum Dis. 2018;77(3):348-54.

36. Liao H, Pan LL, Du J, et al. Efficacy and safety of tocilizumab in patients with Takayasu arteritis. Zhong Hua Nei Ke Za Zhi. 2019;58(6):444-8 (in Chinese). 